\title{
Effect of IrMn inserted layer on anomalous-Hall resistance and spin-Hall magnetoresistance in $\mathrm{Pt} / \mathrm{IrMn} / \mathrm{YIG}$ heterostructures
}

T. Shang', H. L. Yang, Q. F. Zhan' , Z. H. Zuo, Y. L. Xie, L. P. Liu, S. L. Zhang, Y. Zhang, H. H. Li, B. M. Wang, Y. H. Wu, S. Zhang', and Run-Wei Li'

Citation: J. Appl. Phys. 120, 133901 (2016); doi: 10.1063/1.4964114

View online: http://dx.doi.org/10.1063/1.4964114

View Table of Contents: http://aip.scitation.org/toc/jap/120/13

Published by the American Institute of Physics

\section{Articles you may be interested in}

Effect of $\mathrm{NiO}$ inserted layer on spin-Hall magnetoresistance in $\mathrm{Pt} / \mathrm{NiO} / \mathrm{YIG}$ heterostructures

J. Appl. Phys. 109, 032410032410 (2016); 10.1063/1.4959573

Perspective: Interface generation of spin-orbit torques

J. Appl. Phys. 120, 180901180901 (2016); 10.1063/1.4967391

\section{A|P| Jounal of Applied Physics}

INTRODUCING INVITED PERSPECTIVES

Ultrafast magnetism and THz spintronics

Authors: Jakob Walowski and Markus Münzenberg 


\title{
Effect of IrMn inserted layer on anomalous-Hall resistance and spin-Hall magnetoresistance in $\mathrm{Pt} / \mathrm{IrMn} / \mathrm{YIG}$ heterostructures
}

\author{
T. Shang, ${ }^{1, a)}$ H. L. Yang, ${ }^{1}$ Q. F. Zhan, ${ }^{1, \text { b) }}$ Z. H. Zuo, ${ }^{1}$ Y. L. Xie, ${ }^{1}$ L. P. Liu, ${ }^{1}$ S. L. Zhang, ${ }^{1}$ \\ Y. Zhang, ${ }^{1}$ H. H. Li, ${ }^{1}$ B. M. Wang, ${ }^{1}$ Y. H. Wu, ${ }^{2}$ S. Zhang, ${ }^{3, c)}$ and Run-Wei $\mathrm{Li}^{1, \mathrm{~d})}$ \\ ${ }^{1}$ Key Laboratory of Magnetic Materials and Devices and Zhejiang Province Key Laboratory of Magnetic \\ Materials and Application Technology, Ningbo Institute of Material Technology and Engineering, \\ Chinese Academy of Sciences, Ningbo, Zhejiang 315201, China \\ ${ }^{2}$ Department of Electrical and Computer Engineering, National University of Singapore, 4 Engineering Drive 3, \\ Singapore 117583 \\ ${ }^{3}$ Department of Physics, University of Arizona, Tucson, Arizona 85721, USA
}

(Received 9 August 2016; accepted 21 September 2016; published online 4 October 2016)

\begin{abstract}
We report an investigation of anomalous-Hall resistance (AHR) and spin-Hall magnetoresistance (SMR) in $\mathrm{Pt} / \mathrm{Ir}_{20} \mathrm{Mn}_{80} / \mathrm{Y}_{3} \mathrm{Fe}_{5} \mathrm{O}_{12}$ (Pt/IrMn/YIG) heterostructures. The AHR of Pt/IrMn/YIG heterostructures with an antiferromagnetic inserted layer is dramatically enhanced as compared to that of the Pt/YIG bilayer. The temperature dependent AHR behavior is nontrivial, while the IrMn thickness dependent AHR displays a peak at an IrMn thickness of $3 \mathrm{~nm}$. The observed SMR in the temperature range of $10-300 \mathrm{~K}$ indicates that the spin current generated in the Pt layer can penetrate the IrMn layer $(\leq 3 \mathrm{~nm})$ to interact with the ferromagnetic YIG layer. The lack of conventional anisotropic magnetoresistance (AMR) implies that the insertion of the IrMn layer between Pt and YIG could efficiently suppress the magnetic proximity effect (MPE) on induced Pt moments by YIG. Published by AIP Publishing. [http://dx.doi.org/10.1063/1.4964114]
\end{abstract}

\section{INTRODUCTION}

Antiferromagnets (AFMs) are promising candidates for spintronic applications. ${ }^{1}$ Compared to ferromagnetic (FM) materials, the AFMs exhibit unique advantages, e.g., zero net magnetization, insensitivity to the external magnetic perturbation, lack of stray field, and access to extremely high frequency. Recently, the generation and transmission of spin current in AFMs have attracted great attention. The spin pumping and spin Seebeck effect studies on $(\mathrm{Pt}, \mathrm{Ta}) /(\mathrm{NiO}$, $\mathrm{CoO}) / \mathrm{Y}_{3} \mathrm{Fe}_{5} \mathrm{O}_{12}$ (YIG) heterostructures demonstrate that the spin current generated in YIG layer can pass through the antiferromagnetic (AFM) insulator $\mathrm{NiO}$ or $\mathrm{CoO}$ layer and can be detected in the Pt or Ta layer by the inverse spin-Hall effect (ISHE). ${ }^{2-6}$ Similar results were also revealed in (Pt, Ta)/IrMn/CoFeB or $\mathrm{Pt} / \mathrm{NiO} / \mathrm{FeNi}$ heterostructures by the spin-torque ferromagnetic resonance (ST-FMR) technique, where the spin current generated by the spin-Hall effect (SHE) in the Pt or Ta layer can propagate through the IrMn or NiO layer and change the FMR linewidth. ${ }^{7-9}$ The spin current generated by the spin pumping or spin Seebeck effect was also observed in $\mathrm{IrMn} / \mathrm{YIG}, \mathrm{Cr} / \mathrm{YIG}$, and $X \mathrm{Mn} / \mathrm{Py}$ $(X=\mathrm{Fe}, \mathrm{Pd}, \mathrm{Ir}$, and $\mathrm{Pt})$ bilayers through the ISHE. ${ }^{10-14}$ Moreover, the $\mathrm{Pt} / \mathrm{Cr}_{2} \mathrm{O}_{3}$ and $\mathrm{Pt} / \mathrm{MnF}_{2}$ bilayers exhibit a large ISHE voltage, implying that the AFMs can be both the spincurrent detector and generator. ${ }^{15,16}$ These investigations

\footnotetext{
a) Present address: Swiss Light Source and Laboratory for Scientific Developments and Novel Materials, Paul Scherrer Institut, CH-5232 Villigen PSI, Switzerland.

b)Electronic mail: zhanqf@nimte.ac.cn

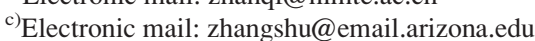

${ }^{\mathrm{d})}$ Electronic mail: runweili@nimte.ac.cn
}

open up new opportunities in developing the AFM-based spin-current devices.

The IrMn alloy, which has been widely used to pin an adjacent FM layer in spin-valve devices via the exchange bias effect, ${ }^{17}$ demonstrates a large ISHE voltage when in contacts with YIG. ${ }^{10}$ Recently, a large SHE and an anomalousHall effect (AHE) have been theoretically proposed in $\mathrm{Cr}$, FeMn, and IrMn AFMs owing to their large spin-orbit coupling (SOC) or Berry phase of the non-collinear spin textures. $^{18-20}$ The experimental investigation of the AHE and SHE on the AFMs could be helpful from both fundamental and practical viewpoints for AFM spintronics. In this work, we investigate the AHE and SHE in $\mathrm{Pt} / \mathrm{Ir}_{20} \mathrm{Mn}_{80} / \mathrm{Y}_{3} \mathrm{Fe}_{5} \mathrm{O}_{12}$ (Pt/IrMn/YIG) heterostructures by measuring the anomalousHall resistance (AHR) and spin-Hall magnetoresistance (SMR). Our findings suggest that the AHR can be dramatically enhanced by inserting an IrMn layer between Pt and YIG, and the spin current generated in the Pt layer can penetrate the IrMn layer to interact with the YIG layer.

\section{EXPERIMENTAL METHODS}

The $\mathrm{Pt} / \mathrm{IrMn} / \mathrm{YIG}$ heterostructures were prepared on (111)-orientated $\mathrm{Gd}_{3} \mathrm{Ga}_{5} \mathrm{O}_{12}$ (GGG) substrates in a combined ultra-high vacuum $\left(10^{-9}\right.$ Torr) pulse laser deposition (PLD) and sputter system. The high quality epitaxial YIG films were deposited via the PLD technique as described elsewhere. $^{21,22}$ The IrMn and Pt films were subsequently sputtered in an in situ process in a 4 mTorr argon atmosphere at room temperature. The thickness and crystal structure of the prepared films were characterized by a Bruker D8 Discover high-resolution $\mathrm{x}$-ray diffractometer (HRXRD). The surface topography of the films was measured in a Bruker Icon 
atomic force microscope. The FMR was measured by electron spin resonance spectrometers. The measurements of transverseHall resistance, longitudinal resistance, and magnetization were carried out in a Quantum Design physical properties measurement system (PPMS) with a rotation option and magnetic properties measurement system (MPMS), respectively.

\section{RESULTS AND DISCUSSION}

Figure 1(a) plots a representative room-temperature XRD patterns for an epitaxial YIG/GGG film near the (444) reflections. As shown in Fig. 1(b), no indication of impurities or misorientation was detected in the range of $20^{\circ}-80^{\circ}$. Clear Laue oscillations indicate a good flatness and uniformity of the prepared film. In this study, the thicknesses of YIG and Pt films, determined by the simulation of x-ray reflectivity (XRR) spectra, are approximately 60 and $3 \mathrm{~nm}$, respectively, while the IrMn thickness ranges from 0 to $8 \mathrm{~nm}$. The atomic force microscope surface topography of the $\mathrm{Pt} / \mathrm{IrMn}(3) / \mathrm{YIG}$ heterostructure over an area of $5 \mu \mathrm{m}$ $\times 5 \mu \mathrm{m}$ in Fig. 1(c) reveals a root-mean-square surface roughness of $0.18 \mathrm{~nm}$, indicating that the prepared films are atomically flat (The number in the brackets represents the thickness of the IrMn layer in $\mathrm{nm}$ ). A representative FMR derivative absorption spectrum of the YIG film shown in Fig. 1(d) exhibits a peak-to-peak value of $\Delta \mathrm{H}=8 \mathrm{Oe}$, which was measured at a radio frequency of $9.39 \mathrm{GHz}$ and a power of $0.1 \mathrm{~mW}$ with an in-plane magnetic field at room temperature. The estimated damping constant $\alpha=2.1 \times 10^{-3}$ is comparable with the previously reported value. ${ }^{23}$

As shown in the top panel of Fig. 2, in order to measure the transverse-Hall and longitudinal resistance, all the
Pt/IrMn/YIG heterostructures were patterned into the Hallbar configuration (central area: $0.3 \mathrm{~mm} \times 10 \mathrm{~mm}$; electrode $0.3 \mathrm{~mm} \times 1 \mathrm{~mm}$ ) by using a shadow mask during the growth. The transverse-Hall resistance $\mathrm{R}_{\mathrm{xy}}$ was measured in the temperature range of $10-300 \mathrm{~K}$ with perpendicular magnetic field ranging from -70 to $70 \mathrm{kOe}$. The AHR is obtained by subtracting the ordinary-Hall resistance (OHR) from the measured $R_{x y}$ up to high field, i.e., $R_{A H R}=R_{x y}-R_{O H R}$ $\times \mu_{0} H$. Figures 2(d) and 2(e) present the resulting $\mathrm{R}_{\mathrm{AHR}}$ as a function of magnetic field for Pt/YIG and Pt/IrMn(1)/YIG, respectively. It is noted that the nonmagnetic Pt becomes magnetic when in contacts with the FM YIG due to its proximity to the Stoner FM instability, i.e., magnetic proximity effect (MPE), as previously shown experimentally by x-ray magnetic circular dichroism (XMCD) and theoretically by first principles calculation. ${ }^{24,25}$ The magnetized Pt shares some common features as the magnetic YIG film, e.g., magnetic anisotropy. ${ }^{21}$ Thus, when the magnetic field approaches zero, the magnetic field dependent $\mathrm{R}_{\mathrm{AHR}}$ exhibits an M-shaped behavior close to zero field. However, for Pt/IrMn/ YIG, the $\mathrm{R}_{\mathrm{AHR}}$ continuously decreases as approaching zero field, implying that both interfaces of Pt/IrMn and IrMn/YIG are free of MPE, which is consistent with the absence of conventional anisotropic magnetoresistance (AMR) (see below). The derived anomalous-Hall resistivity $\rho_{\text {AHR }}$ is summarized as functions of temperature [Fig. 2(f)] and IrMn thickness $\left(t_{\text {IrMn }}\right)$ [Fig. 2(g)], respectively. The $\rho_{\text {AHR }}$ for all the Pt/IrMn/ YIG exhibits complicated temperature dependence. Both the magnitude and sign are highly non-trivial, which were also found in $\mathrm{Pt} / \mathrm{LaCoO}_{3}$ bilayers. ${ }^{26}$ The $\rho_{\mathrm{AHR}}$ changes its sign near the temperature of $50 \mathrm{~K}$ that is almost independent of the IrMn thickness. Moreover, as increasing the $t_{\mathrm{IrMn}}$, the $\rho_{\mathrm{AHR}}$
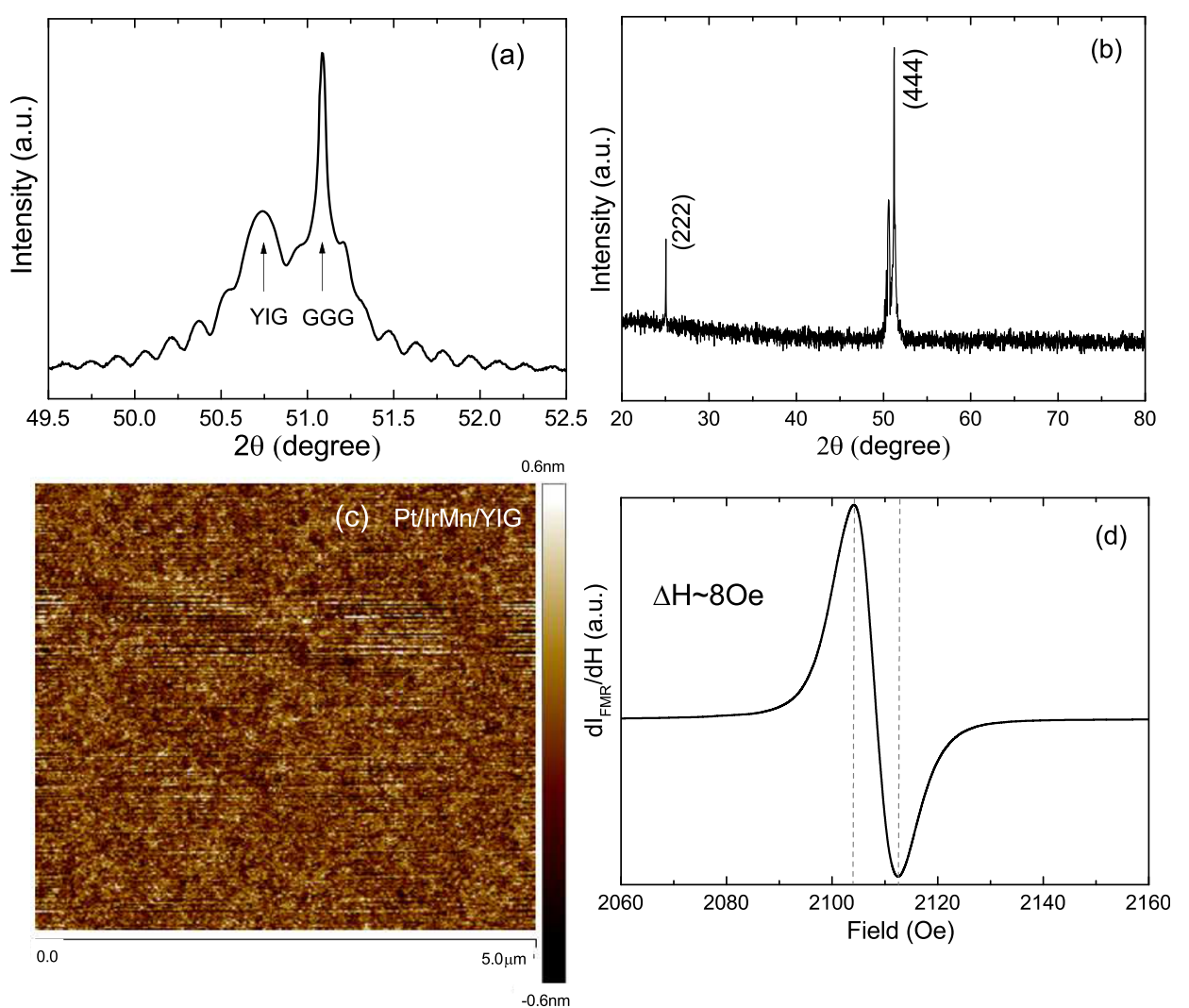

FIG. 1. The representative 20- $\omega$ XRD patterns for a YIG/GGG film (a) near the (444) reflections and (b) from $20^{\circ}$ to $80^{\circ}$. (c) Atomic force microscope surface topography of the $\mathrm{Pt} / \mathrm{IrMn}(3) /$ YIG heterostructure over an area of $5 \mu \mathrm{m} \times 5 \mu \mathrm{m}$. (d) A FMR derivative absorption spectrum of a $60 \mathrm{~nm}$ YIG film. 

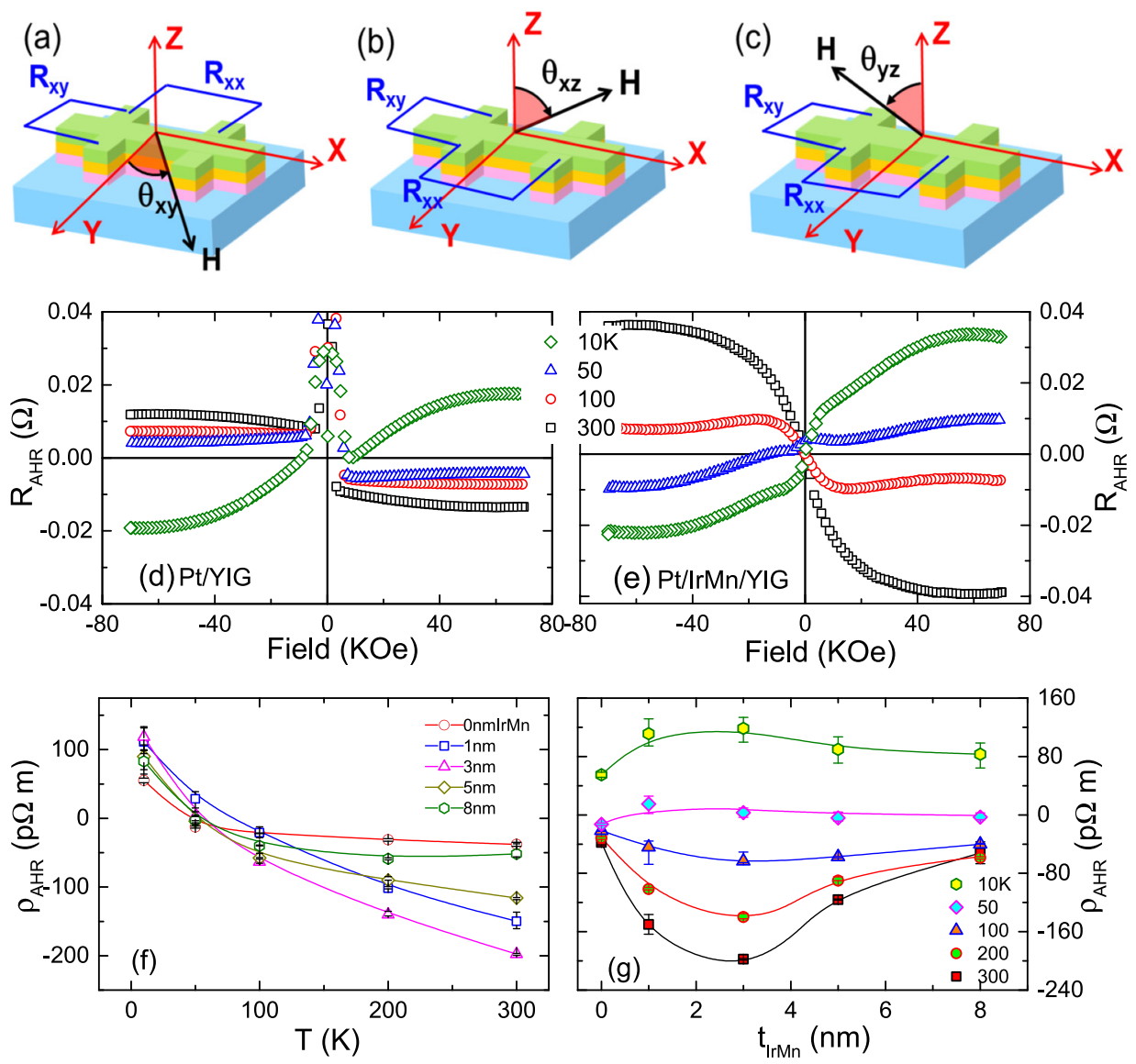

FIG. 2. (a)-(c) Schematic plots of the longitudinal and transverse Hall resistance measurements. The magnetic field orientations $\theta_{\mathrm{xy}}, \theta_{\mathrm{xz}}$, and $\theta_{\mathrm{yz}}$ are with respect to the $\mathrm{y}^{-}, \mathrm{z}_{-}$, and $\mathrm{z}$-axes, respectively. The electric current is applied along the $x$-axis. AnomalousHall resistance $R_{A H R}$ for (d) Pt/YIG and (e) $\mathrm{Pt} / \mathrm{IrMn}(1) / \mathrm{YIG}$ as a function of magnetic field at different temperatures. (f) Temperature dependence of the $\rho_{\text {AHR }}$ for $\mathrm{Pt} / \mathrm{IrMn} / \mathrm{YIG}$ with various IrMn thicknesses. The $\rho_{\text {AHR }}$ is replotted versus IrMn thickness at various temperatures in $(\mathrm{g})$. All $\rho_{\text {AHR }}$ values are averaged by $\left[\rho_{\mathrm{AHR}}(70 \mathrm{kOe})-\rho_{\mathrm{AHR}}\right.$ (-70 kOe)]/2. increases and reaches a maximum around $t_{\mathrm{IrMn}}=3 \mathrm{~nm}$, which excludes the interfacial MPE origin of the observed AHR.

The AMR for Pt/IrMn/YIG was also measured down to low temperatures. As an example, the AMR of Pt/YIG and $\mathrm{Pt} / \mathrm{IrMn}(1) / \mathrm{YIG}$ for three different field scans is presented in Fig. 3. When the magnetic field scans within the xy plane [Figs. 3(a) and 3(d)], both the conventional AMR and SMR contribute to the total AMR; for the Xz scan [Figs. 3(b) and $3(\mathrm{e})]$, the resistance changes are attributed to the MPEinduced conventional AMR; for the $y z$ scan [Figs. 3(c) and 3(f)], the conventional AMR is zero, and only the SMR is expected. ${ }^{27,28}$ As shown in Fig. 3(e), the resistance is almost independent of $\theta_{\mathrm{xz}}$, indicating an extremely weak MPE at the interfaces of $\mathrm{Pt} / \mathrm{IrMn}$ or $\mathrm{IrMn} / \mathrm{YIG}$. In contrast, the MPE is significant at the Pt/YIG interface [see Fig. 3(b)]: the maximum amplitude of conventional AMR is around $2.2 \times 10^{-4}$, which is comparable to that of SMR. Since the conventional AMR is negligible in Pt/IrMn(1)/YIG, the SMR dominates the AMR when the magnetic field is varied within the $x y$ plane, the amplitudes of the $\theta_{x y}$ scan are almost identical to those of the $\theta_{\mathrm{yz}}$ scan. While for Pt/YIG, due to the MPEinduced conventional AMR, none of the amplitudes is identical to each other. The AMR amplitudes for $\theta_{\mathrm{xy}}, \theta_{\mathrm{xz}}$, and $\theta_{\mathrm{yz}}$ scans are summarized as a function of temperature in Figs. 3(g) and 3(h) for Pt/YIG and Pt/IrMn(1)/YIG, respectively. Upon decreasing the temperature, the SMR persists down to $10 \mathrm{~K}$, with the amplitudes monotonically decreasing from $7.5 \times 10^{-5}(300 \mathrm{~K})$ to $3.0 \times 10^{-5}(10 \mathrm{~K})$ in $\mathrm{Pt} / \mathrm{IrMn}(1) / \mathrm{YIG}$. Due to the smaller spin-Hall angle, the shorter spin diffusion length, and the larger electrical resistivity of IrMn, the SMR amplitudes of Pt/IrMn/YIG are almost an order smaller than that of Pt/YIG. ${ }^{10,13,27}$ The temperature dependence of SMR amplitudes for $\mathrm{Pt} / \mathrm{IrMn} / \mathrm{YIG}$ is significantly different from the Pt/YIG or Pd/YIG bilayers, where the SMR amplitudes exhibit a nonmonotonic temperature dependence and acquire a maximum around $100 \mathrm{~K} .{ }^{29,30}$ For Pt/YIG, the temperature dependence of SMR can be described by a single spinrelaxation mechanism. ${ }^{29}$ The spin diffusion length is defined as $\lambda=\sqrt{D \tau_{\mathrm{sf}}}$, where $D$ and $\tau_{\mathrm{sf}}$ are the diffusion constant and the spin-flip relaxation time, respectively. Within the ElliotYafet spin-orbit scattering model, both $D$ and $\tau_{\text {sf }}$ are inversely proportional to the resistivity. ${ }^{31,32}$ In the Pt metal, the electrical resistivity mainly originates from the phononelectron scattering $(\rho \propto T)$ at high temperature, then $\lambda \propto$ $1 / T$. However, when the extra magnetic-electron scattering needs to be considered in $\mathrm{Pt} / \mathrm{IrMn} / \mathrm{YIG}$ heterostructures, the assumption of $\lambda \propto 1 / T$ is no longer valid. Moreover, the spin diffusion length of IrMn is comparable to the electron mean free path; thus, the Elliot-Yafet mechanism is likely no longer available. On the other hand, as suggested by the theoretical calculation, the spin current propagates in AFMs in terms of diffusive thermal magnon. ${ }^{33}$ The magnon thermal number decreases with decreasing temperature, which may provide the explanation of the temperature dependence of the SMR shown in Fig. 3(h). It is noted that the Pt/IrMn(3)/ YIG also exhibits a SMR with the amplitude of $6.8 \times 10^{-5}$ at room temperature, while there is no SMR signal in $\mathrm{Pt} / \mathrm{IrMn}(5) / \mathrm{YIG}$, implying that the spin current generated in 

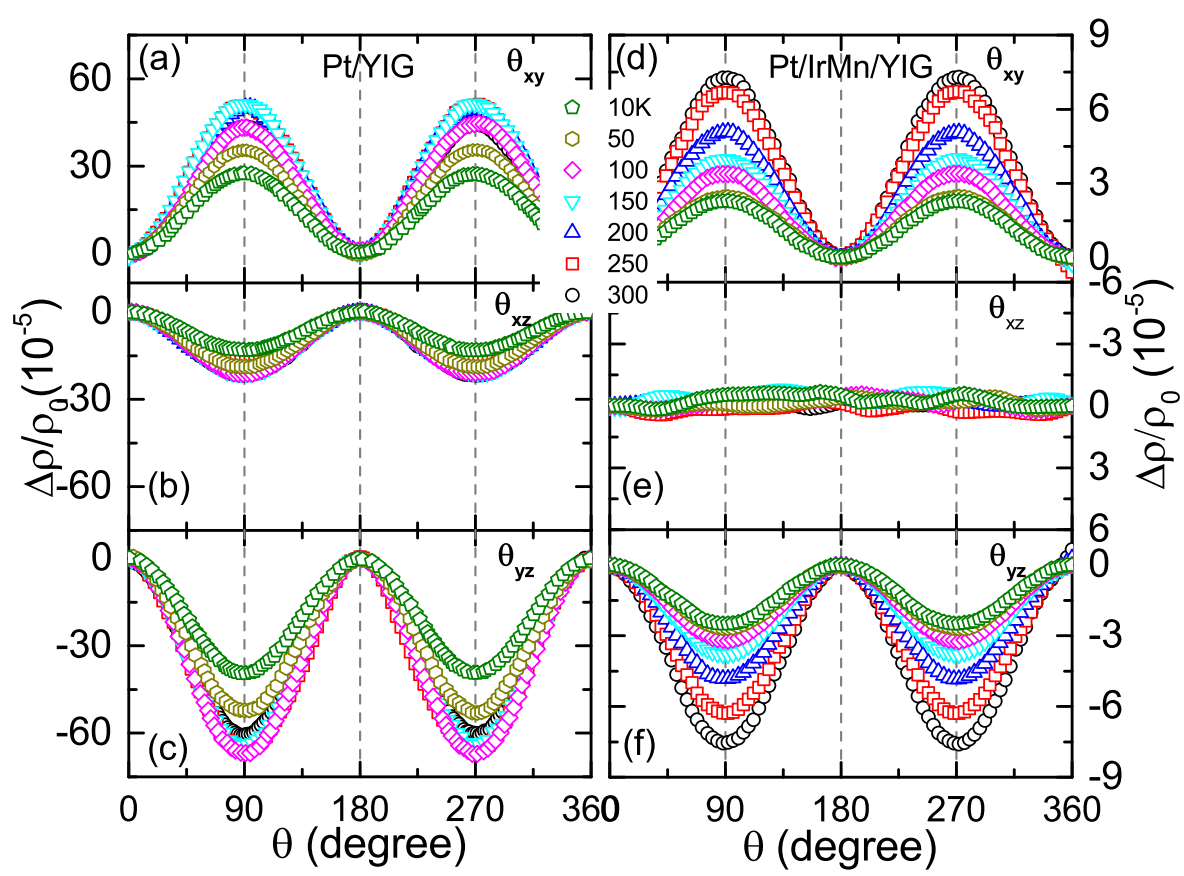

FIG. 3. Anisotropic magnetoresistance at various temperatures for (a)-(c) $\mathrm{Pt} /$ YIG and (d)-(f) Pt/IrMn(1)/YIG with magnetic field varied within $x y, x z$, and yz planes. Temperature dependence of AMR amplitudes for Pt/YIG and $\mathrm{Pt} / \mathrm{IrMn}(1) / \mathrm{YIG}$ heterostructures are plotted in $(\mathrm{g})$ and $(\mathrm{h})$, respectively. The cubic, circle, and triangle symbols stand for the $\theta_{\mathrm{xy}}, \theta_{\mathrm{xz}}$, and $\theta_{\mathrm{yz}}$ scans, respectively.

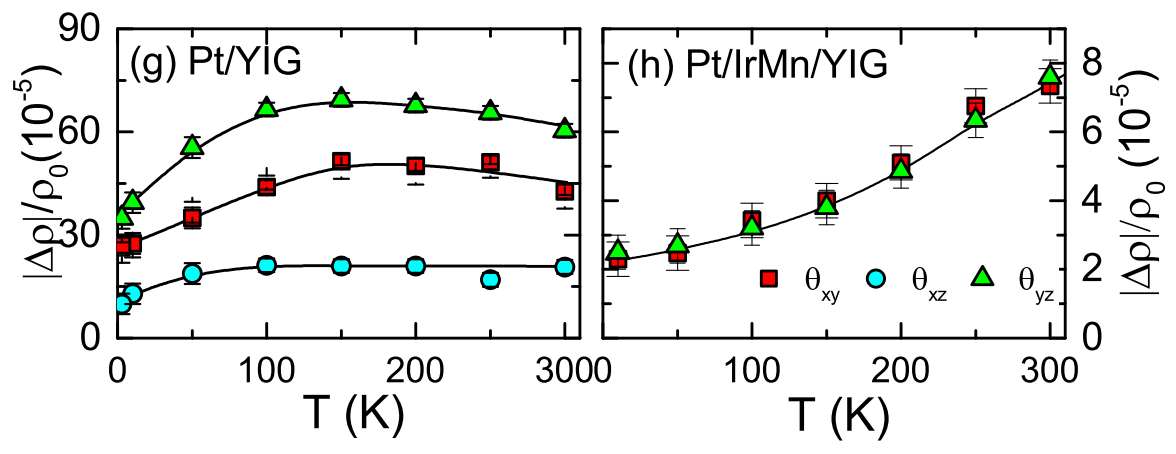

the Pt can penetrate the IrMn layer $(\leq 3 \mathrm{~nm})$ to interact with the FM YIG layer.

The magnetic ordering temperature for a very thin AFM film is expected to be well below the corresponding transition temperature of bulk forms. In order to track the magnetic ordering temperature of IrMn, we measured the exchange bias field $H_{\mathrm{e}}$ for Pt/IrMn/YIG heterostructures. As an example, the typical normalized magnetic hysteresis loops $M / M_{\mathrm{s}}$ for $\mathrm{Pt} / \mathrm{IrMn}(1) / \mathrm{YIG}$ at different temperatures are presented in Fig. 4(a). The derived $H_{\mathrm{e}}$ versus temperature is summarized in Fig. 4(b). The onset of exchange bias field $H_{\mathrm{e}}$ is defined as the blocking temperature, as indicated in Fig. 4(b). The estimated $T_{\mathrm{b}}$ for 1,3 , and $5 \mathrm{~nm}$ IrMn are approximately around 180,200 , and $230 \mathrm{~K}$, respectively. It is noted
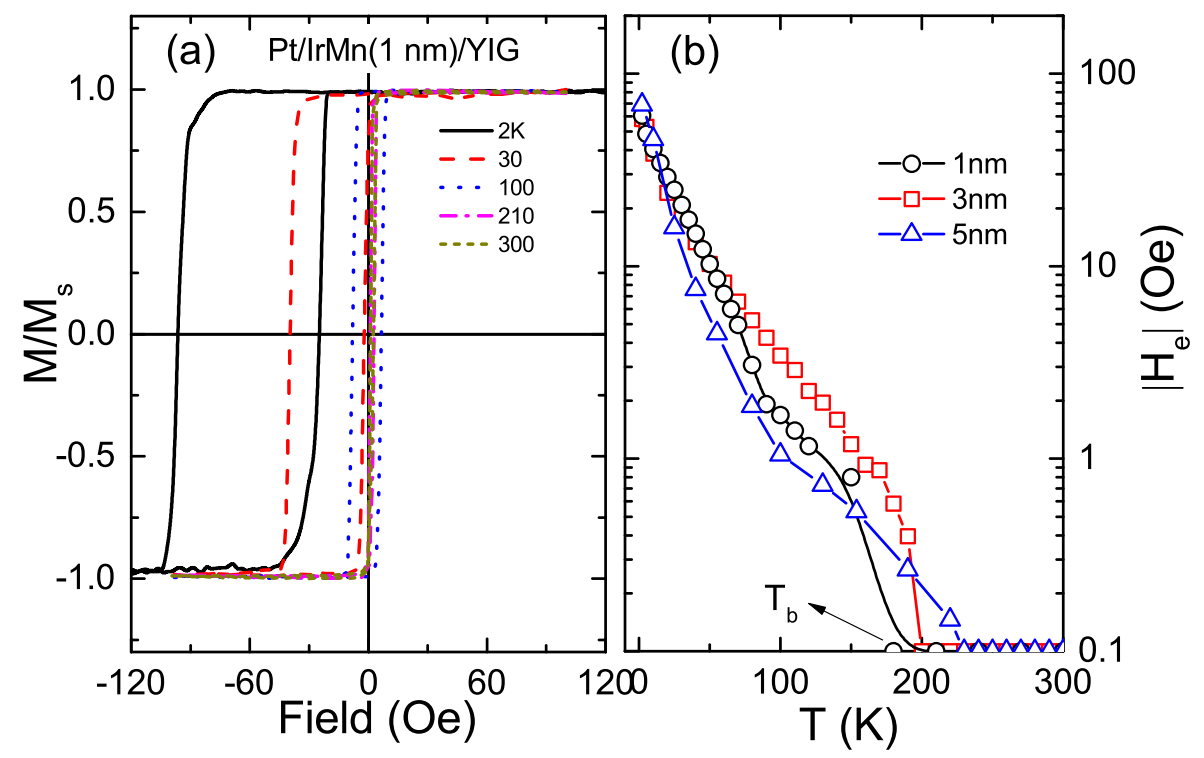

FIG. 4. (a) Normalized hysteresis loops for $\mathrm{Pt} / \mathrm{IrMn}(1) / \mathrm{YIG}$ at various temperatures. (b) Temperature dependent $H_{\mathrm{e}}$ for $\mathrm{Pt} / \mathrm{IrMn} / \mathrm{YIG}$ heterostructures with different IrMn thicknesses. 
that the exchange bias field does not monotonically increase with the IrMn thickness and shows the largest value with $3 \mathrm{~nm} \mathrm{IrMn}$, which was previously found in other IrMn-based bias systems. ${ }^{34,35}$

Based on the above experimental results, we discuss the origins of the significant AHR in $\mathrm{Pt} / \mathrm{IrMn} / \mathrm{YIG}$ heterostructures. There are at least four contributions to the observed AHR in Pt/IrMn/YIG: the MPE, the spin-Hall based SMR, the spin-dependent interface scattering, and the intrinsic properties of the IrMn metal. In contrast to the Pt/YIG, the absence of conventional AMR in $\mathrm{Pt} / \mathrm{IrMn} / \mathrm{YIG}$ indicates the negligible MPE at Pt/IrMn or IrMn/YIG interfaces. The SMR model also predicts an anomalous-Hall-like resistance, ${ }^{27}$ whose magnitude and sign are determined by the spin diffusion length and spin-Hall angle of the metal and the imaginary part of the spin mixing conductance, respectively. Though the SMR model can describe the thickness dependence of $\mathrm{AHR}$ in $\mathrm{Pt} / \mathrm{IrMn} / \mathrm{YIG}$, it fails to explain the temperature dependent AHR by the following reasons: (i) An arbitrary temperature dependence of the imaginary part of the spin mixing conductance parameter is required to qualitatively describe the temperature dependent AHR including the sign change; (ii) According to the spin pumping studies, both the spin-Hall angle and the spin diffusion length of IrMn are smaller than those of Pt, which cannot explain the enhancement of AHR with increasing the IrMn thickness. ${ }^{10,13,14}$ The spin-dependent scattering at the interface, combined with the conventional skew-scattering and side-jump mechanisms, can also contribute to the AHR. ${ }^{36}$ However, the enhancement of AHR with IrMn thickness excludes the interfacial origin. Finally, the large AHE and SHE in the IrMn metal predicted by the previous theoretical calculations are attributed not only to the large SOC of heavy Ir atoms but also to the Berry phase of the non-collinear spin structures of IrMn. ${ }^{18-20}$ Such AHR induced by the non-collinear magnetism could be related to the magnon diffusion length of IrMn, which may exhibit a maximum value when the IrMn thickness is comparable to its magnon diffusion length. In order to qualitatively explain this nontrivial temperature dependent behavior, further theoretical and experimental investigations are highly desirable.

Now we discuss the possible interplay between the AFM order and the spin transport properties, including the SMR and AHR. As shown in Figs. 2 and 3, there is no clear discrete change in AHR or SMR near the blocking temperatures of the IrMn layer, implying a weak relationship between the AHE or SHE and the AFM order in IrMn. Similar results were also observed in $\mathrm{Cr} / \mathrm{YIG}$ bilayers, where both the ISHE voltage and AHR are independent of the AFM ordering temperature. ${ }^{12}$ According to our magnetic characterizations (Fig. 4), the AFM ordering temperatures of IrMn films are well below room temperature. However, the enhancement of AHR in $\mathrm{Pt} / \mathrm{IrMn} / \mathrm{YIG}$ as compared to that in Pt/YIG happens in the whole studied temperature range [see Fig. 2(g)]. There are two possible reasons for such a phenomenon. The AHE and SHE due to the non-collinear magnetism happen in a length scale of nanometer, which is a local property and is independent of the long range magnetic order, as reported previously in the $\mathrm{Mn}_{5} \mathrm{Si}_{3}$ film. ${ }^{37}$ Another reason is that the strength of
SOC is independent of the AFM order in the IrMn metal, which is mainly contributed from the heavy Ir atoms.

\section{CONCLUSION}

In summary, we report an investigation of the AHE and SHE by measuring the AHR and SMR in Pt/IrMn/YIG heterostructures. The significantly enhanced AHR in Pt/IrMn/YIG is likely associated with the strong SOC and non-collinear magnetic structure of IrMn, and the sizable SMR indicates that the spin current can transport through IrMn. The observed non-trivial temperature dependence of AHR cannot be consistently explained by the existing theories, which demands further theoretical and experimental investigations.

\section{ACKNOWLEDGMENTS}

We thank the high magnetic field laboratory of Chinese Academy of Sciences for the FMR measurements. This work was financially supported by the National Natural Science foundation of China (Grants Nos. 11274321, 11404349, 51502314, 51522105, and 11374312) and the Key Research Program of the Chinese Academy of Sciences (Grant No. KJZD-EW-M05). S. Zhang was partially supported by the U.S. National Science Foundation (Grant No. ECCS1404542).

${ }^{1}$ A. H. MacDonald and M. Tsoi, Philos. Trans. R. Soc., A 369, 3098 (2011).

${ }^{2}$ C. Hahn, G. de. Loubens, O. Klein, M. Viret, V. V. Naletov, and J. B. Youssef, Europhys. Lett. 108, 57005 (2014).

${ }^{3}$ H. L. Wang, C. H. Du, P. C. Hammel, and F. Y. Yang, Phys. Rev. Lett. 113, 097202 (2014).

${ }^{4}$ Z. Qiu, J. Li, D. Hou, E. Arenholz, A. T. NDiaye, A. Tan, K. Uchida, K. Sato, Y. Tserkovnyak, Z. Q. Qiu, and E. Saitoh, Nat. Commun. 7, 12670 (2016).

${ }^{5}$ W. Lin, K. Chen, S. Zhang, and C. L. Chien, Phys. Rev. Lett. 116, 186601 (2016).

${ }^{6}$ A. Prakash, J. Brangham, F. Yang, and J. P. Heremans, Phys. Rev. B 94, 014427 (2016).

${ }^{7}$ T. Moriyama, M. Nagata, K. Tanaka, K.-J. Kim, H. Almasi, W. Wang, and T. Ono, e-print arXiv:1411.4100.

${ }^{8}$ H. Reichlová, D. Kriegner, V. Holý, K. Olejník, V. Novák, M. Yamada, K. Miura, S. Ogawa, H. Takahashi, T. Jungwirth, and J. Wunderlich, Phys. Rev. B 92, 165424 (2015).

${ }^{9}$ T. Moriyama, S. Takei, M. Nagata, Y. Yoshimura, N. Matsuzaki, T. Terashima, Y. Tserkovnyak, and T. Ono, Appl. Phys. Lett. 106, 162406 (2015).

${ }^{10}$ J. B. S. Mendes, R. O. Cunha, O. Alves Santos, P. R. T. Ribeiro, F. L. A. Machado, R. L. Rodríguez-Suárez, A. Azevedo, and S. M. Rezende, Phys. Rev. B. 89, 140406(R) (2014).

${ }^{11}$ L. Frangou, S. Oyarzún, S. Auffret, L. Vila, S. Gambarelli, and V. Baltz, Phys. Rev. Lett. 116, 077203 (2016).

${ }^{12}$ D. Qu, S. Y. Huang, and C. L. Chien, Phys. Rev. B 92, 020418(R) (2015).

${ }^{13}$ W. Zhang, M. B. Jungfleisch, W. Jiang, J. E. Pearson, A. Hoffmann, F. Freimuth, and Y. Mokrousov, Phys. Rev. Lett. 113, 196602 (2014).

${ }^{14}$ J. Sinova, S. O. Valenzuela, J. Wunderlich, C. H. Back, and T. Jungwirth, Rev. Mod. Phys. 87, 1213 (2015) and reference therein.

${ }^{15}$ S. Seki, T. Ideue, M. Kubota, Y. Kozuka, R. Takagi, M. Nakamura, Y. Kaneko, M. Kawasaki, and Y. Tokura, Phys. Rev. Lett. 115, 266601 (2015).

${ }^{16}$ S. M. Wu, W. Zhang, K. C. Amit, P. Borisov, J. E. Pearson, J. S. Jiang, D. Lederman, A. Hoffmann, and A. Bhattacharya, Phys. Rev. Lett. 116, 097204 (2016).

${ }^{17}$ K. Hoshino, R. Nakatani, H. Hoshiya, Y. Sugita, and S. Tsunashima, Jpn. J. Appl. Phys. 35, 607 (1996). 
${ }^{18}$ H. Chen, Q. Niu, and A. H. MacDonald, Phys. Rev. Lett. 112, 017205 (2014).

${ }^{19}$ R. Shindou and N. Nagaosa, Phys. Rev. Lett. 87, 116801 (2001).

${ }^{20}$ F. Freimuth, S. Blügel, and Y. Mokrousov, Phys. Rev. Lett. 105, 246602 (2010).

${ }^{21}$ T. Shang, Q. F. Zhan, H. L. Yang, Z. H. Zuo, Y. L. Xie, H. H. Li, L. P. Liu, B. M. Wang, Y. H. Wu, S. Zhang, and R.-W. Li, Sci. Rep. 5, 17734 (2015).

${ }^{22}$ T. Shang, Q. F. Zhan, H. L. Yang, Z. H. Zuo, Y. L. Xie, H. H. Li, L. P. Liu, S. L. Zhang, B. M. Wang, Y. H. Wu, S. Zhang, and R.-W. Li, Appl. Phys. Lett. 109, 032410 (2016).

${ }^{23}$ M. C. Onbasli, A. Kehlberger, D. H. Kim, G. Jakob, M. Kläui, A. V. Chumak, B. Hillebrands, and C. A. Ross, APL Mater. 2, 106102 (2014).

${ }^{24}$ Y. M. Lu, Y. Choi, C. M. Ortega, X. M. Cheng, J. W. Cai, S. Y. Huang, L. Sun, and C. L. Chien, Phys. Rev. Lett. 110, 147207 (2013).

${ }^{25}$ Q. Qu, S. Y. Huang, J. Hu, R. Q. Wu, and C. L. Chien, Phys. Rev. Lett. 110, 067206 (2013)

${ }^{26}$ T. Shang, Q. F. Zhan, H. L. Yang, Z. H. Zuo, Y. L. Xie, Y. Zhang, L. P. Liu, B. M. Wang, Y. H. Wu, S. Zhang, and R.-W. Li, Phys. Rev. B 92 165114 (2015).
${ }^{27}$ Y. T. Chen, S. Takahashi, H. Nakayama, M. Althammer, S. T. B. Goennenwein, E. Saitoh, and G. E. W. Bauer, Phys. Rev. B 87, 144411 (2013).

${ }^{28}$ M. Isasa, A. B. Pinto, S. Vélez, F. Golmar, F. Sánchez, L. E. Hueso, J. Fontcuberta, and F. Casanova, Appl. Phys. Lett. 105, 142402 (2014).

${ }^{29}$ S. R. Marmion, M. Ali, M. McLaren, D. A. Williams, and B. J. Hickey, Phys. Rev. B 89, 220404(R) (2014).

${ }^{30}$ T. Lin, C. Tang, H. M. Alyahayaei, and J. Shi, Phys. Rev. Lett. 113, 037203 (2014).

${ }^{31}$ J. Fabian and S. Das Sarma, Phys. Rev. Lett.81, 5624 (1998).

${ }^{32}$ L. Q. Liu, R. A. Buhrman, and D. C. Ralph, e-print arXiv:1111.3702.

${ }^{33}$ S. M. Rezende, R. L. Rodríguez-Suárez, and A. Azevedo, Phys. Rev. B 93, 054412 (2016).

${ }^{34}$ M. Ali, C. H. Marrows, M. Al-Jawad, and B. J. Hickey, Phys. Rev. B 68, 214420 (2003).

${ }^{35}$ Ch.Gritsenko, I. Dzhun, N. Chechenin, G. Babaytsev, and V. Rodionova, Phys. Procedia 75, 1066 (2015).

${ }^{36}$ N. Nagaosa, J. Sinova, S. Onoda, A. H. MacDonald, and N. P. Ong, Rev. Mod. Phys. 82, 1539 (2010) and reference therein.

${ }^{37}$ C. Sürgers, W. Kittler, T. Wolf, and H. v. Loehneysen, AIP Advances 6, 055604 (2016). 